\title{
Influence of the Compressible Flexographic Sleeve Exploitation on Print Quality
}

\author{
Saša PETROVIĆ* ${ }^{*}$ Nemanja KAŠIKOVIĆ, Dragoljub NOVAKOVIĆ, Gordana BOŠNJAKOVIĆ, Emine ARMAN KANDIRMAZ, Arif OZCAN
}

\begin{abstract}
Due to the nature of the used materials, the quality of the flexographic print depends on a large number of process parameters and the materials parameters in all the phases of the production. During the pre-press, sleeves and stickyback tapes are needed for the mounting of the printing forms. These two components in the process of the flexographic printing are the least researched ones, even though they have a potentially significant impact on the stability and the quality of the printing process, as well as the print quality itself. Therefore, this research is oriented towards the investigation of the influence of the compressible sleeve exploitation on the process and print quality parameters through the measurement of optical density, tone value increase and trapping. The aim of the research is the improvement of stability and predictability of the printing process and print quality through the defining of the intensity and the trends of changes in densitometric parameters of quality.
\end{abstract}

Keywords: densitometry; exploitation; flexographic sleeve; print quality; printing process stability

\section{INTRODUCTION}

Flexography is a process of relief printing in the sense that the image areas of the printing form are raised above the non-image areas. Due to the use of flexible printing forms, a very light contact pressure is sufficient in order to secure proper ink transfer [1]. The process is based on the transfer of the ink from the aniloxroll to the printing (raised) elements of the photopolymer printing form, and from them onto the substrate, at a lowest possible pressure (Kiss Print). Otherwise, printing defects may appear due to factors such as pronounced tone value increase, potentially leading to deformation of the printing elements.

\subsection{Print Quality Parameters}

Assessment of print quality is generally a challenging undertaking. Choosing measurable parameters of quality control depends on the intended use of the final product and the user's expectations. The quality parameters are controlled both during the processing and the final control. The most typically measured parameters in the course of the processing and final control are the optical density, trapping and the tone value increase (TVI).

The researchers who evaluate the impact made by the variables on the print quality most typically choose one or more print quality parameters which they intend to observe for changes. Their focus is mainly set on the parameters of the optical density, contrast, gloss, TVI and ink layer uniformity.

\subsubsection{Optical Density}

Optical density is a significant parameter in all printing techniques. As the layer of ink on the surface increases, so does the optical density, moving from the 0 value on a surface with no ink at all, to the maximum $D_{\infty}$ value of optical density, whereupon there is no further growth of the value with the further increase of the ink film thickness [2].

\subsubsection{Tone Value Increase}

TVI is the inevitable incidence in halftone areas which occurs during the course of printing. The TVI should never be compensated in full, due to the nature of the human eye, with its inherent expectation of a certain TVI [3].

Deformation of halftone dots on the printing form occurs due to two mechanisms. The first is the expansion of the dot surface area, occurring as a result of the pressure. The intensity of this deformation is determined by the Poisson ratio $(v)$. The second mechanism known as the dot barrelling is the deformation defined by the lateral walls of the printing elements which become partly added to the surface of the elements in the course of the pressure-aided transfer of ink. At a certain pressure, the level of dot barrelling becomes determined by the Young's modulus of elasticity of the photopolymer printing form. This type of deformation is practically non-existent in rigid material with a high Young's modulus. The curve of TVI due to dot barrelling shows that an increase in tone values in highlight areas causes a sudden drop in the share of dot barrelling in the overall increase of tone values, owing to the structural strength gained by the halftone dots on the printing forms. However, upon entering the area of midtones, the share of dot barrelling in the TVI is on the rise again, due to the increase in circumference of the dots [4]. These two occurrences, together with the spreading of ink due to the differences in free surface energies of the substrate and ink, are the main factors in determining the mechanical TVI [5].

Another type of TVI occurring upon each measuring of the properties via the share of the reflected light is the optical TVI. As a result of the light dispersion, a certain proportion remains trapped under the halftone dot or absorbed by the ink, and the other reflects around the edges of the dot which leads to the optical growth of the dot area. Based on their research presented in paper [6], the authors established that the greater the penetration of ink, the lesser the optical TVI.

\subsubsection{Trapping}

Trapping is defined as the capability of the ink to adhere to the layer of previously applied printing ink [7]. Good adherence of the ink is dependent on rheological features (viscosity and tackiness) of the ink being printed 
over, as well as on the thickness of the ink film and the ink sequence in the course of printing.

One of the defects on the prints which is often found in overprinted inks is manifested in the form of channels or smaller or bigger holes in the layer of the printed ink. At higher speeds of printing, destabilisation occurs at the contact surface of the air and the ink in the printing NIP, causing defects. In paper [8], the authors classified this printing instability within a larger group of hydrodynamic defects, which was described by Saffman and Taylor, whose name it still holds. The Saffman-Taylor instability occurs when a less viscous fluid (in the given case, the air) passes through the contact zone in the more viscous fluid (ink, in the given case), forming in the process channels in the applied layer of ink.

\subsection{Influence of Process and Materials Parameters on Print Quality}

Due to the nature of the used materials, the quality of the flexographic print depends on a large number of process parameters and the materials parameters in all the phases of the production.

During the pre-press, sleeves and stickyback tapes are needed for the mounting of the printing forms. These two components in the process of the flexographic printing are the least researched ones, even though they have a potentially significant impact on the stability and the quality of the printing process, as well as the print quality itself. Their most important properties include composition, structure, thickness, thickness variations, resilience, temperature stability, compressibility and adhesive features.

The researchers studied the impact of the majority of process and materials parameters, keeping the other parameters constant.

\subsubsection{Mounting Materials}

Based on the research conducted in paper [4], the mechanical deformation of the printing forms during printing, as well as the TVI are dependent on two characteristics of photopolymer material, the Poisson ratio and Young's modulus.

The Young's modulus is a dynamic property which increases with the increase in the printing NIP pressure or during cyclic loading, meaning that the stress-induced hardening occurs in the material [4]. This mechanical property leads to a reduction in the intensity of deformation, thereby alleviating the TVI [9].

In order to obtain a high print quality, thinner printing forms are mounted either using stickyback tapes with a compressible layer, or regular stickyback tapes in combination with sleeves that already have a compressible layer. Therefore, the hardness of a printing form must be observed in combination with the sleeve and the stickyback tape, since these elements have a changing impact on the elasticity of the entire system $[9,10]$. Having conducted research in paper [11], the author concluded that under exposure to the pressure of $1 \mathrm{MPa}$, a sleeve with a compressible layer undergoes a deformation of $9 \%$, while the deformation of the harder and softer printing forms is in the range of around $1 \%$. This means that the deformation of the halftone dot element in contact with the surface is reduced to a minimum.

Using the experimental approach in paper [12] the author reached a conclusion that a slightly harder stickyback tape undergoes lesser compression when exposed to pressure. This means that the printing form must become more deformed in contact with the substrate. An increase in the contact zone leads to the increase in spreading of the ink in the printing NIP. As opposed to this, the softer stickyback tape enables greater relaxation of the printing form through greater compression of the compressible layer of the tape. This is an important factor in the printing of halftone areas since it enables avoidance of distortion and enables a sharper printed halftone dot.

The importance of the sleeve characteristics in determining the print quality was highlighted in the research conducted by the authors in paper [13]. Using the experimental method, the authors made a conclusion that among the studied parameters (ink, sleeve type, the length of the print run), it was the sleeve characteristics that made the most significant impact on the TVI. Namely, the sleeves with the compressible foam of the medium level of hardness recorded a significantly larger TVI, especially in highlights, compared with sleeves with a softer compressible layer or the stickyback tape mounted onto a hard sleeve.

One of the typical occurrences in flexographic printing easily affected by the mounting materials is bouncing. Bouncing is a printing problem in flexography wherein the plate cylinder physically loses contact with anilox or impression cylinder at some points [14].

The drop of the resilience and occurrence of stress induced hardening take away from the ability of the sleeves and plates to absorb energy. According to [15], if left unabsorbed, this energy could cause bouncing. At the same time, according to [16] bouncing occurs when cylinders lose contact with each other during printing. This could happen due to the larger drop in resilience of the sleeves, leading to less pronounced returning of the sleeves to the original diameter.

\subsubsection{Pressure}

Many innovations in flexographic technology are directed towards printing pressures. According to the paper [17], densitometric values mostly impacted by the pressure variations are dot area, dot shape and tone gradation. Higher pressure values may lead to ink spreading at the edges of printing areas. The higher the pressure, the more prone are the halftone elements to deformation and ink spreading, resulting in the TVI. Research into the impact made by pressure on the print performed on OOP material proved that the pressure between the printing form and the impression cylinder has the greatest impact on the reproduction of dot areas with a significant increase in the optical density [18]. In their research paper [19] the authors recorded that the highest TVI actually occurred in the areas with the coverage under $3 \%$, due to the collapse of the halftone dot elements. The author of paper [20] studied the contact between the hard and soft cylinders in the printing NIP, reaching a conclusion that the largest 
impact on the pressure profile was made by the thickness of the compressible elastomer layer and the width of the contact area. Considering the fact that a high-quality print requires low pressure, this means that all of the changes in the eccentricity and deviation in diameter of the sleeve or the impression cylinder pose the need to increase the pressure, in order to alleviate their impact on the print. This leads to deformities and other types of incidence which cause TVI. In order to reduce this increase in the pressure, it is necessary to transfer the load from the printing forms onto the compressible layer of the sleeves or the stickyback tapes. In this way, the deformation of the printing elements is alleviated, as well as the spreading of the ink caused by the higher pressure.

In their experiment in paper [21] the authors have shown that the ink transfer increases with the increase in the pressure, while it declines with the increase of the speed. The pressure between the printing form and the substrate has an impact on the roughness of porous substrates. Higher pressure forces tend to compress and flatten the network of fibres, thereby increasing the contact area of the ink and the substrate. The increase of the pressure will also lead to lateral spreading of the ink on the surface and, in the case of porous substrates, penetration into the substrate by means of capillary absorption [21-23].

An increase in pressure in the printing NIP results in a better ink transfer aided by a large number of different mechanisms [18, 21]. In paper [22], the authors conducted a research into the impact made by pressure on the printing process which revealed that the percentage of ink transferred from the printing form will increase with the increase of pressure, whereas the authors of paper [3] came to a conclusion that the increase in the ink transfer at high pressures stops after a certain moment.

\subsubsection{Ink Viscosity}

The viscosity of the ink and the surface tension have an impact on the transfer of ink and its adhesion to the substrate [24]. The lower the ink viscosity, the higher the chances for its spreading over the surface of non-porous printing substrates or penetration into the structure of porous printing substrates $[22,23]$. The decline in the ink viscosity is mostly due to the thinning caused by shear forces as a result of high printing speeds [25], but it may also occur as a result of an increase in temperature. For example, the authors of the paper [26] pointed out that a temperature increase of $5.5^{\circ} \mathrm{C}$ can reduce the viscosity of a water based printing ink by $50 \%$ or more. In the paper [23] the authors also provide a brief discussion on the impact of the vehicle viscosity on the ink transfer, stating that a lower viscosity of the vehicle apparently improves the transfer of the ink. However, this observation is not fully supported by the research.

The author of the paper [27] states that an increase in the ink viscosity results in a decreased impact of the printing speed and the pressure. He also pointed out that a higher level of the ink viscosity means a lower degree of penetration of the ink into the substrate, even though this process is largely impacted by the porous nature of the substrate. As far as the ink penetration is concerned, he concluded that this parameter was increasing in parallel with the increase of the ink quantity on the printing form, but only to a certain degree, i.e. up to the moment when the substrate becomes saturated with ink.

The impact made by the difference in ink viscosity on trapping is demonstrated indirectly, through the fast drying of the ink applied to the substrate, resulting in the trapping values more typical of the process of wet-on-dry printing, which is better than trapping in wet-on-wet printing.

\subsubsection{Temperature}

The authors of papers [28, 29] studied the rheological characteristics of ink exposed to changes in temperature, reaching a conclusion that the response follows the Arrhenius equation. In addition to this, in paper [29] the authors detected the difference in the behaviour of inks with different pigments in which the cyan ink is considerably more sensitive to temperature variations than black. By increasing the temperature, there is an impact on the ink and its characteristics that plays an important role in the quality of the print, the viscosity and the surface tension [30]. Regarding the ink viscosity, in paper [31] the authors established that it decreases with the increase in the temperature, with a simultaneous increase in the optical density. In the printing NIP, the pressure-driven penetration of the vehicle into the substrate is easier, causing partial immobilisation of the ink. With the viscosity declining with the increase in the temperature, the ease of evaporation of the water component during drying is on the rise. Faster evaporation results in thicker layers of immobilised ink in the printing NIP [32]. Since the separation of the ink film occurs in the immobilised area, the increase in its thickness definitely promotes the enhanced transfer of the ink, which explains the increase in the optical density.

Regarding the TVI, in paper [31] the authors observed a decline in its value with the increase of the temperature. They concluded that the explanation for this trend lies in the fact that a reduction in the viscosity of ink occurring at a higher temperature enables easier absorption of the vehicle into the substrate and a faster bonding and immobilisation of the ink layer, thereby preventing its spreading across the surface of the substrate. In addition to this, fast evaporation enhances faster immobilisation, which in turn contributes to the reduction in the TVI.

\section{MATERIALS AND METHODS \\ 2.1 Materials and Samples}

For the purposes of the study, Twinlock sleeves of medium hardness rank were used. One set of sleeves was composed of brand new sleeves (further on referred to as $1 \times$ sleeves), whereas the other set included sleeves previously used for 320 times (further on referred to as $320 \times$ sleeves) for the printing of around $5000000 \mathrm{~m}$ of material. The characteristics of the Twinlock sleeves are not precisely defined by the manufacturer. The layers of the sleeve are: the base body of the sleeve, the open-cell polyurethane foam, the stabilisation layer and the self-adhesive tesa Twinlocklayer. According to the sleeves manufacturer's declarations, the sleeves are supposed to be able to provide substitute for Rotec Medium compressible sleeves, 3M stickyback tapes of the 10,13 and 19 series, the Lohmann stickyback tapes of the Duploflex 4 medium, Duploflex 5.2 
and Duploflex 5.3 series, or Tesa stickyback tapes coded 52015 , 52115, 52021, 52121, 52221. Based on the data available for the Rotec Medium sleeves [33], the hardness of the compressible layer of the Twinlock sleeves amounts to 50 Shore A, whereas the data for the Tesa 52015 stickyback tape yields them suitable for the use with 1.14 and $1.7 \mathrm{~mm}$ thick printing forms. Their characteristics are supposed to produce the best results in the event of dot areas and solid areas being on the printing forms which are to be mounted on the same sleeve. During the exploitation, the sleeves were not exposed to the impact of ozone, which may trigger the process of degradation of their qualities.

The sleeves were fitted with nyloflex FTP flat top dot printing forms, manufactured by FlintGroup and developed to special requirements of the Tetra Pak company, where the samples were printed. The characteristics of these printing forms correspond to the commercially available FlintGroupnyloflex FTF Digital printing forms, the difference being a better adaptation to the water-based inks used in printing on absorbent substrates. The changed composition of the polymer in the nyloflex FTP printing forms makes it possible to obtain flat top dots using the standard production procedure, as well as standard devices used in the process of producing conventional digital printing forms. The reason for choosing the printing forms with a flat top is increased control of all process parameters, which in this case occurs through more consistent TVI values due to lesser deformation and compression of the printing elements under pressure. The printing forms were imaged using the ESKO CDI Spark 4260 device with external drum, using the standard AM screening and the circular halftone dots, at the resolution of 54 lines $/ \mathrm{cm}$. The pre-exposure and the main exposure were performed using the Vianord EVO4E device, the development at the Vianord EVO4IP, while the drying and post-exposure were performed using the Vianord EVO4DLF device. The parameters of production of the printing forms are given in Tab. 1.

Table 1 Production parameters of FlintGroupnyloflex FTP printing forms

\begin{tabular}{|l|l|}
\hline Operation & Duration \\
\hline Pre-exposure & $30 \mathrm{~s}$ \\
\hline CTP imaging & $12 \mathrm{~min}$ \\
\hline Main exposure & $660 \mathrm{~s}$ \\
\hline Processing & $190 \mathrm{~mm} / \mathrm{min}$ (cca. $13 \mathrm{~min})$ \\
\hline Drying & $120 \mathrm{~min}$ at $61{ }^{\circ} \mathrm{C}$ \\
\hline Cooling & $10 \mathrm{~min}$ \\
\hline Post-exposure & $2 \mathrm{~min}$ UVA and $5 \mathrm{~min}$ UVC - simultaneously \\
\hline Cutting & cca. $8 \mathrm{~min}$ \\
\hline Resting & $2-3 \mathrm{~h}$ \\
\hline
\end{tabular}

The substrate used for the printing is the $\mathrm{CLC} / \mathrm{C}$ multilayer semi-cardboard coated with calcium carbonate featuring a smooth surface. Its characteristics are shown in Tab. 2. This type of substrate was used in order to avoid non-uniformity of print (in the form of non-printed areas) due to the surface roughness of uncoated substrates.

The inks that were used (cyan and magenta) are PremoNova water-based inks manufactured by Flint, pigments and solvents that are in accordance with the provisions of the Swiss Ordinance 817.023.21 regulation. Their composition includes: Ethyl acetate (5 - 10\%), 1methoxy-2-propanol (<15\%), 1 - propoxypropane-2-ol (1 - $5 \%)$, titanium chelates $(0.25-1 \%)$. In the course of printing, the viscosity of the ink was maintained at $15.5 \mathrm{~m}$
Pas (Ford - 4), and the $\mathrm{pH}$ value at 9, using the system for automatic regulation of viscosity. The inks are dried using penetration and evaporation.

Table 2 Properties of CLC/C semi-cardboard

\begin{tabular}{|l|l|}
\hline Properties & Value \\
\hline Bending force & $315 \mathrm{mN}$ \\
\hline$L^{*}$ colour coordinate & 93 \\
\hline$a^{*}$ colour coordinate & 0 \\
\hline$b^{*}$ colour coordinate & 2.3 \\
\hline Moisture content & $7.4 \%$ \\
\hline Surface weight & $278 \mathrm{~g} / \mathrm{m}^{2}$ \\
\hline Thickness & $420 \mu \mathrm{m}$ \\
\hline Density & $660 \mathrm{~kg} / \mathrm{m}^{3}$ \\
\hline Surface pulling resistance & $292 \mathrm{kPa}$ \\
\hline Surface roughness & $2.25 \mu \mathrm{m}$ \\
\hline
\end{tabular}

The anilox sleeves that were used to apply both the cyan and the magenta ink onto the printing form are manufactured by Zecher. The items in question are the ceramic anilox sleeves featuring the engraving angle of $60^{\circ}$, resolution of 360 lines/cm and volume of hexagonal cells of $3.8 \mathrm{~cm}^{3} / \mathrm{m}^{2}$. The variation of the cell volume measured using the Troika Anicam device at 5 points along the anilox sleeve did not exceed $5 \%$.

The printing of the samples was performed at the VT Flex 175 ES printing press, driven by servo motors.

The press was designed exclusively for the needs of Tetra Pak, in collaboration with the Tresucompany. The printing speed was $600 \mathrm{~m} / \mathrm{min}$, with an even distance of the cylinders through the units, suited to the purpose of maintaining minimum pressure needed for high-quality print, the so-called Kiss Print pressure.

Since the testing was performed at a press designed for industrial-level production, the printing forms were made for a real product, i.e. the Tetra Brik Aseptic packaging of 21 volume and were mounted on $1 \times$ and $320 \times$ Twinlock Medium sleeves. The control patches on which measurements were obtained were added to the areas which are hidden after the packaging is filled and formed. These patches are of a rectangular shape, with the dimension of $6 \times 6 \mathrm{~mm}$, and filled with halftone dots obtained through AM screening and forming the tonal values of $2,5,10,20,30,40,50,70,90 \%$ area coverage. Apart from them, there are two more patches of the same dimensions. One of these patches represents the solid tone of the ink, while the second is generated by over-printing of inks, also in solid tones. The sampling was performed by cutting out the printed material along the width of the roll (from four different points) from the first (1.r), fifth (5.r) and seventh (7.r) roll of the print run (each roll being $4500 \mathrm{~m}$ long), 3 sets of samples per length, both for the print order deploying $1 \times$ sleeves and the print order deploying $320 \times$ sleeves. The number of the samples on which the measuring was performed is 12 samples per roll, which amounts to 36 samples used for measuring the patches for one set of sleeves, i.e. 72 samples for both sets. Expressed in meters of print after reaching the full printing speed, the samples were taken at 300, 18300 and $27300 \mathrm{~m}$ of the print run, at full speed, which corresponds to the beginnings of the 1.r, 5.r and 7.r, respectively.

For the study of the mechanical characteristics under compression, a total of 20 samples were prepared (10 of each type, i.e. 10 for both $320 \times$ and $1 \times$ sleeve) having circular form, $8 \mathrm{~mm}$ diameter and $2 \mathrm{~mm}$ thickness. The samples were 
obtained from various positions along the sleeve length (at each $15 \mathrm{~cm}$ ) and cut using the CNC Aristomat SL 1317 machine. There is no suitable standard for the desired testing using the specified materials, therefore the samples are prepared in accordance with the literature source [34] (with the proportional change of the diameter due to pre-defined thickness which is different), where similar testing was conducted using similar material. Additionally, the surface area and the thickness of the sample correspond to the surface area and thickness of the material part of the ring thermoplastic samples made from similar material following the ISO3384-2005 standard [35].

\subsection{Methods of Measuring}

The information on the values of the optical density, the TVI and trapping were obtained by measuring 12 samples from each roll printed by using the $1 \times$ and $320 \times$ sleeves for the cyan ink. The measuring was done using the $\mathrm{x}$-rite $\mathrm{eXact}$ device, belonging to the group of spectrophotometers. The measuring of optical density and trapping was performed in selected M3 measuring conditions which included the polarisation filter for the purpose of reducing the difference in reflection between the wet and the dry ink, while at the same time the UV section of the radiated light spectrum was excluded. The calibration was carried out at the non-printed part of the substrate.

Calculation of the TV and the TVI based on the optical density was performed using the Murray-Davies formula shown in Eq. (1), whereas the trapping was calculated using the Preucil formula shown in Eq. (2). After completing 12 measuring rounds, their mean value was determined, which is to be used in further presentation, analysis and discussion of results.

$F_{\mathrm{D}}=\left[\frac{\left(1-10^{-D_{\mathrm{R}}}\right)}{\left(1-10^{\left.-D_{\mathrm{V}}\right)}\right.}\right] \cdot 100 \%$

where $D_{\mathrm{R}}$ and $D_{\mathrm{V}}$ represent the optical density of dot areas and solid tone areas, respectively.

$T=\frac{D_{1+2}-D_{1}}{D_{2}}$

where $D_{1+2}$ represents the value of optical density of the areas with over-printed inks, $D_{1}$ being the value of optical density of the solid tone patch of the first and $D_{2}$ the value of optical density of the solid tone patch of the second printed ink. All patches were measured using a filter which is complementary to the second printed ink.

The quantification of the mechanical TVI was performed using software analysis of the images of the patches obtained using the FAG Flex3Pro device with a camera in the accompanying software for analysis. This measuring was performed on the same control patches from the same samples used for the spectrophotometer measurements, in order to gain a realistic insight into the interconnection of the changes to the measured parameters. After taking the images of the fields, these images were analysed using the manual method, based on a selection of
16 halftone dots from each image, arranged within a $4 \times 4$ matrix. The values of parameters obtained in the image analysis were used for the calculation of the mean value which was used during the further presentation, analysis and discussion of the results.

The cyclic compression test of the samples (100 cycles) of the combined polyurethane compressible and adhesive layer of the Twinlock Medium sleeves was performed using the universal testing device Shimadzu Compact Tabletop EZ-LX, at the ambient temperature of $23{ }^{\circ} \mathrm{C}$ and air humidity of $50 \%$. The measuring head with a $2.5 \mathrm{kN}$ threshold was applied, and the strain rate was set to $15 \mathrm{~mm} / \mathrm{min}$. A test of 100 cycles was chosen based on preliminary measurements, where stabilisation of most of the relevant parameters was detected between the 30th and 40th cycle. The samples were placed in between the compression plates with the adhesive layer facing up. The sample orientation is in accordance with the real use pattern, where among the two layers comprising the sample, the adhesive layer is the first one in contact with the pressure suppliers. The maximum strain was set to $25 \%$. The test results were recorded and subsequently processed in the TrapeziumX software, where the stressstrain curves were also plotted.

\section{RESULTS}

\subsection{Mechanical Properties}

The residual strain, measured using a cyclic compression test of 100 cycles corresponds to the strain measured at the minimum stress point of the relaxation curve occurring at the moment of transition to a new cycle. Fig. 1 shows the measurings recorded during the first three cycles, and then following each third cycle. The first three cycles are recorded because it is during them that the most significant changes occur, i.e. the most substantial difference occurs between the first and the second cycle, followed by a period of stabilisation.

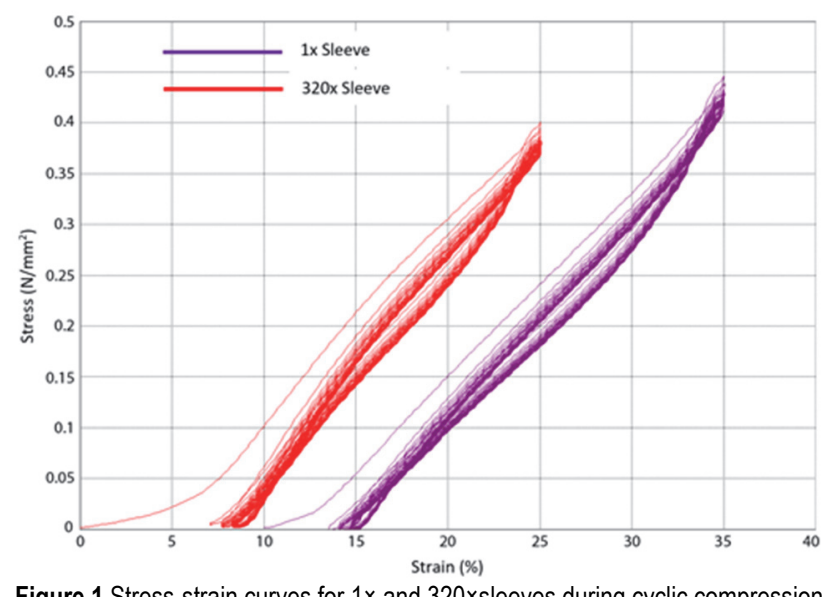

Figure 1 Stress-strain curves for $1 \times$ and $320 \times$ sleeves during cyclic compression test

The trend of change is mostly the same for the sleeves in both age groups-however, individual values show almost a double difference. The percentage of the residual strain in the case of $320 \times$ sleeves is almost $50 \%$ higher already after the second cycle, compared to the residual strain of $1 \times$ sleeve in 99 th cycle. 
On the other hand, the maximum stress of the $1 \mathrm{x}$ sleeves is slightly more than $10 \%$ higher than the maximum stress for the same cycle for the $320 \times$ sleeves. This initial difference in the maximum stress at the set maximum strain is kept through all of the cycles.

\subsection{Tone Value Increase}

The TVI curves for the cyan samples printed using $1 \times$ and $320 \times$ sleeves are shown in Fig. 2. A few observations can be made:

- TVI for $320 \times-7 . r$ is the biggest for all TV, except for $50 \%$;

- TVI for $1 \times-1 . r$ is the smallest or among the smallest for all TV, except for $50 \%$ and $90 \%$;

- In the highlight areas, the TVI curves for the samples printed from $320 \times$ sleeves correspond to the higher values; - TVI of samples printed from $1 \times$ sleeve in highlights and mid-tone area rises with the number of roll;

- TVI of samples printed from $320 \times$ sleeve in highlights and mid-tone area also rises with the number of roll;

- The largest divergence is present on the $2 \% \mathrm{TV}$ patches at the 7 . r. The values of the samples printed from the $320 \times$ sleeves indicate a significant TVI, which then drops again; - The TVI in the highlight and midtone areas are such that the $320 \times-5$.r curve follows or demonstrates only slightly higher values compared to the $1 \times-7 . r$ curve;

- If $2 \% \mathrm{TV}$ patch is excluded, the regularity of TVI curves slightly increases with an increase in the number of printed rolls and exploitation of the sleeves. Namely, the 1x curves become more regular after printing of the 5.r, while with $320 \times$ curves this happens before printing of the 5.r;

- In the midtones area $(50 \% \mathrm{TV})$ there is a noticable decrease of dispersion of the results;

- For the 50\% TV there is also noticable decrease in the values of TVI starting from the $320 \times-5 . r$ and $1 \times-7 . r$;

- Upon entering the darker shadow areas, the dispersion of the TVI for all of the samples is on the decline.

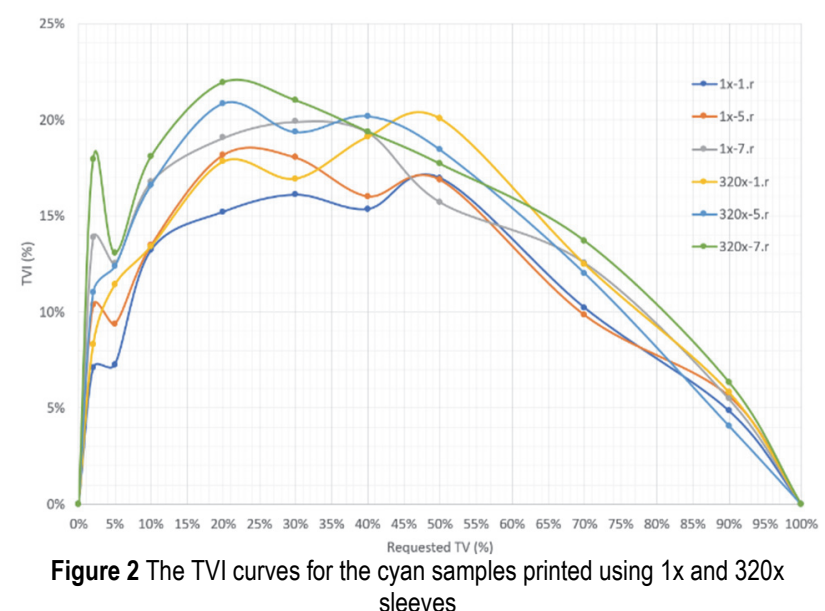

When observing Fig. 3 showing the TVI at the most critical patches $(2,5,10$ and $50 \%$ TV) for the $1 \times$ and $320 \times$ sleeves it becomes apparent that the most significant TVI was measured for the $2 \%$ TV patch on $320 \times$ - 7.r sample, whereas the decline in TVI occurring with the increase of the number of the printed roll was observed only at the $50 \% \mathrm{TV}$ patch for $1 \times-7 . \mathrm{r}$, and $320 \times-5 . \mathrm{r}$, with the retention of relatively constant values on the $320 \times-7 . r$ as well.

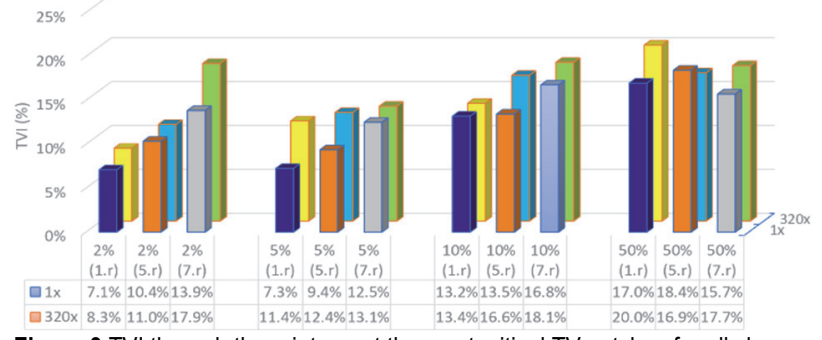

Figure 3 TVI through the print run at the most critical TV patches for all sleeves

The differences in the TVI between all of the samples can be observed in. Fig. 3 as well. The TVI is more significant in the case of the $320 \times$ sleeves, with the exception of the $50 \%$ TV patch, for both 1.r and 7.r.

The presentation of the measured mechanical TVI per roll for the critical patches of the cyan ink, printed using the $1 \times$ and $320 \times$ sleeves, is given in Fig. 4 . The tendency of TVI is present both for the $1 \times$ and the $320 \times$ sleeves; however, it tends to be less pronounced with the $320 \times$ sleeves except for $2 \%$ TV for $320 \times-7$.r. Divergence from the trend is present only in the $50 \% \mathrm{TV}$ patch for $1 \times-7 . \mathrm{r}$, $320 \times-5 . r$ and $320 \times-7 . r$.

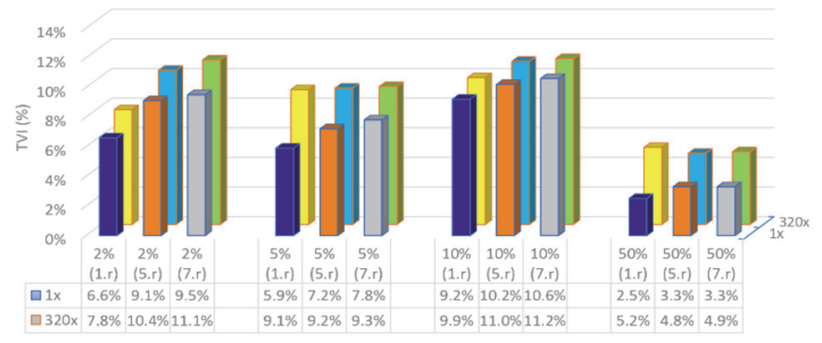

Figure 4 Mechanical TVI through the print run at the most critical TV patches for all sleeves

In the Fig. 4 it can be observed that the difference in mechanical TVI between $1 \times$ and $320 \times$ sleeves becomes smaller with the increase of the number of printed rolls, except for $2 \%$ TV for $320 \times-7$.r. In other words, although initially significantly larger than their correspondents in the $1 \times$ sleeves, the mechanical TVI for $320 \times$ sleeves shows greater stability and slower growth.

\subsection{Optical Density}

The results in measuring the optical density of the cyan solid tone patches for all of the samples are shown in Fig. 5 . There is a noticeable trend of the growing change in optical density, occurring with the increase in the number of the printed roll.

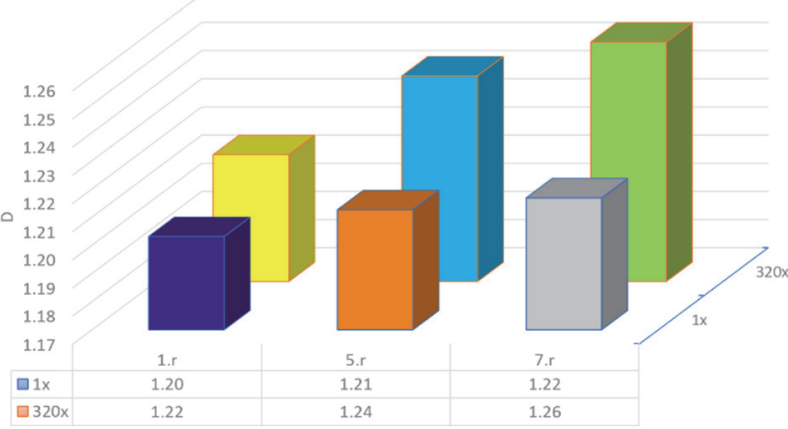

Figure 5 Optical densities of solid tone patches through the print run for all sleeves 
The solid tone patch ought to be observed as the patch with the most representative indication of the change in the application of the ink layer. The relations between the optical density for the solid tone patches can also be observed in Fig. 5 , where higher values correspond to the samples printed using the $320 \times$ sleeves, whereas the differences in optical density between imprints rendered by the $1 \times$ and $320 \times$ sleeves are smaller in the case of the 1.r, compared to the ones obtained from the 7.r.

\subsection{Trapping}

The values of trapping measured at the secondary blue colour patch (obtained by over-printing the magenta over the cyan ink) for all of the samples are shown in Fig. 6. The values of trapping tend to grow in parallel with the increase in the number of printed rolls, as well as the difference in favour of the $320 \times$ sleeves. The exception to this are the samples taken from the 1.r, where the values measured at the samples obtained using the $320 \times$ sleeves, are slightly if not insignificantly lower than the values of trapping measured on the samples rendered by the $1 \times$ sleeves.

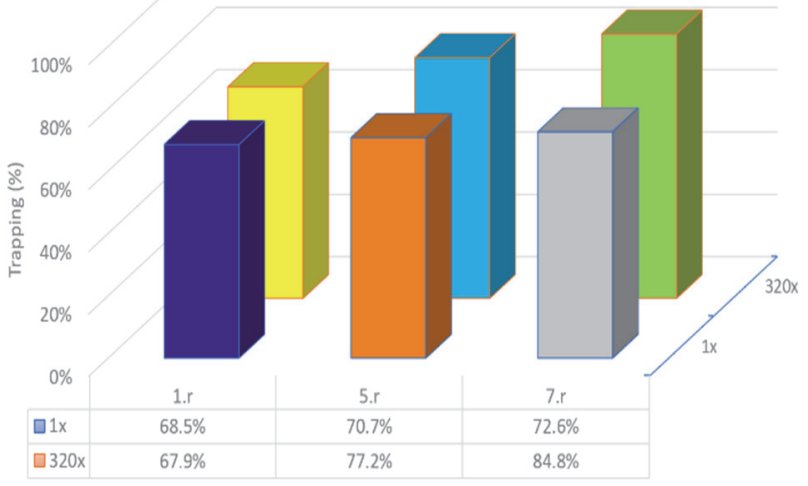

Figure 6 Trapping values through the print run at the secondary blue colour patch printed using $1 \times$ and $320 \times$ sleeves

Fig. 7a and Fig. 7b show magnified images of the patches of the secondary blue colour, taken from the $1 \times-$ 1.r and $320 \times-1 . r$ samples, while Fig. $8 \mathrm{a}$ and Fig. 8 b show the same patches taken from the $1 \times-7 . r$ and $320 \times-7 . r$ samples. In the case of the 1.r, the nonuniformities on the surface of the patches are relatively even, regardless of the type of sleeve used. However, in the samples taken from the 7.r, the nonuniformities are visually less expressed in samples obtained using the $320 \times$ sleeves.

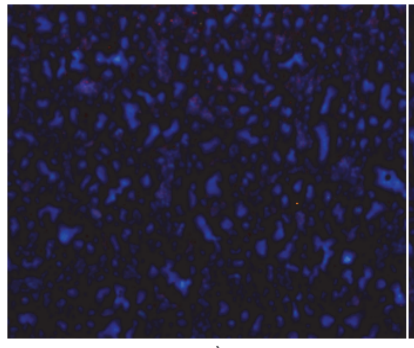

a)

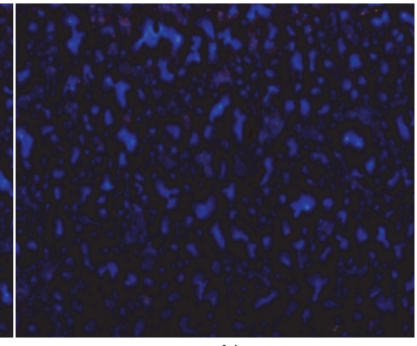

b)
Figure 7 Magnified image of the secondary blue colour patch printed on 1.r using a) $1 \times$; b) $320 \times$ sleeves

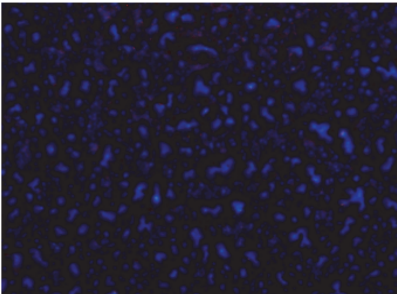

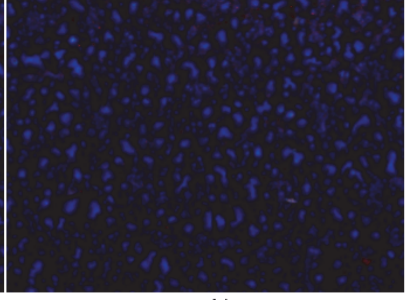

Figure 8 Magnified image of the secondary blue colour patch printed on 7.r using a) $1 x$; b) $320 x$ sleeves

\section{DISCUSSION}

\subsection{Tone Value Increase}

The observed difference in TVI between the prints made using the $1 \times$ and $320 \times$ sleeves is most pronounced in highlight areas due to the fact that the printing elements in highlight areas are most prone to deformation which leads to the TVI. Since the printing elements which correspond to the $2 \% \mathrm{TV}$ are produced as individual ones, without the support provided by the walls of neighbouring elements, the increase in the load placed on the flexible printing form due to the higher residual strain of the $320 \times$ sleeves compressible layer leads to a higher deformation of the printing elements. This is represented by the significant TVI, which is reduced and stabilised after the transition to areas of $5 \%$ and $10 \% \mathrm{TV}$, due to the increase in the stability of the printing elements.

The difference in the mechanical TVI caused by the number of the printed rolls is mostly slightly higher in the case of the $1 \times$ sleeves (except for $2 \%$ TV for $7 . r$ ), due to the changes in the mechanical characteristics of the sleeve, which require more time to reach stable values, compared to already partially fatigued material of the $320 \times$ sleeves, whose changes in the mechanical qualities induced at the start of the print remain largely constant irrespectively of the number of the printed rolls.

The difference in the speed of reaching the maximum values of residual strain between the $1 \times$ and $320 \times$ sleeves is easily noticed at the $50 \% \mathrm{TV}$ patches. At the $50 \% \mathrm{TV}$, a reduction in TVI occurs due to the stress-induced hardening of the printing elements in flexible printing forms, thereby reducing their deformation (while the lateral expansion grows, the usually more pronounced dot barrelling declines) which leads to the mechanical TVI. To make this hardening possible, the printing forms must undergo a series of cyclic loadings. In the $1 \times$ sleeves, this change occurs on the 7.r, which is an indicator of the fact that the stable value of the residual strain for the $1 \times$ sleeve was reached between the printing of the 6.r and 7.r. In the $320 \times$ sleeves, hardening of the printing elements occurred earlier, i.e. prior to the printing of the 5.r, meaning that the maximum residual strain in the $320 \times$ sleeves was already reached between the 2.r and 4.r. The cause of the delay in hardening of the printing elements in the forms mounted on the $1 \times$ sleeves lies in the insufficient loading intensity and number of cycles, whereas in the case of the $320 \times$ sleeves the reason for the delay is mostly due to the insufficient number of cycles, and only to a lesser extent to the insufficient loading intensity.

A less pronounced difference between the changes caused by the drop in the resilience of the different sleeves in the shadow areas is present due to the lesser available 
space for the changes and increased mechanical stability of the printing elements.

The change of the TVI in the highlight areas shows a growing trend, occurring due to the change in the resilience of the sleeve compressible layer, followed by the increase in the load placed on the printing elements of the printing forms, as well as their wear. There are no signs of the divergence from the growing trend of TVI in highlight areas. Namely, the hardening of the polymer following the increase in the intensity of the cyclic deformation of the printing elements due to the change in the sleeve resilience is not capable of making a significant impact on the deformation mechanisms of relatively isolated printing elements, as is the case on the ones found in 50\% TV patches.

The differences between the mechanical TVI coinciding with the increase in the number of the printed rolls are smaller in the case of $320 \times$ sleeves, despite the fact that the values themselves are higher than the ones for the samples printed using $1 \times$ sleeves. The reason for this lies in the fact that the final change in resilience and the residual strain of $320 \times$ sleeves occurs much faster compared to the $1 \times$ sleeves, which in itself allows for generally higher predictability of the printing process and the output quality in the case of $320 \times$ sleeves.

However, a large discrepancy between mechanical and total TVI is observed in the patches of $2 \% \mathrm{TV}$, where the difference of the TVI values between $1 \times$ and $320 \times$ sleeves shows no reduction, but rather a large increase. This divergence is caused by the occurrence of major vibrations and bouncing of the sleeve with the printing forms, leading to a doubling of the halftone dots, and eventually to a significant TVI. The pronounced vibrations and the doubling are caused by a large drop in the resilience and stressinduced hardening. Due to this, there is a weakening of the quality of compensation of the vibration caused by sudden transitions from the printing to the non-printing elements and channels created during the mounting of the printing forms onto the sleeves.

The smaller difference in the mechanical TVI on the prints obtained using $320 \times$ sleeves is further enhanced by the faster drying of the ink on the substrate, thereby reducing its spreading under the pressure of the printing elements. The drying of the ink is faster due to the heat released through the more pronounced hysteresis loss for the $320 \times$ sleeves, reducing the viscosity of the ink. This, in turn, enables better penetration of the ink into the substrate, and a faster drying process, lowering the possibility of the ink spreading over a larger area.

A greater drop in the TVI for the $320 \times$ sleeves, measured at the $50 \% \mathrm{TV}$ patch is due to the fast occurrence of the change in resilience, exposing the printing elements to higher loads very early, i.e. with the printing of the 1.r. This leads to a higher TVI since stress-induced hardening of polymers requires time. However, once the hardening is completed, the drop in the TVI is more pronounced than in the case of $1 \times$ sleeves, where the degradation in resilience progresses gradually. This is further enhanced by the previously explained drop in the ink viscosity. The gradual degradation of the resilience in $1 \times$ sleeves means that the drop in the TVI will not precede its growth, which occurs due to the gradual increase in the deformation of the printing elements, prior to the hardening of the polymers.

\subsection{Optical Density}

On the solid tone patch, the increase of the optical density occurring in parallel with the increase in the number of printed rolls is attributed to the drop in the resilience, i.e. to the increase in hardness and load placed on the printing form. This is in accordance with the fact that the harder stickyback tapes are used in the cases when it is necessary to print larger surfaces of solid tone, while at the same time the softer tapes are typically used for printing of the fine halftone areas.

The difference in the optical density of the solid tone patches in favour of the $320 \times$ sleeves is in accordance with the speed of change of the resilience and the residual strain for $1 \times$ and $320 \times$ sleeves, as well as with the fact that a reduction in the viscosity of the thin film of ink on the printing form results in the increase in transfer and faster penetration and evaporation of the vehicle, thus creating a thicker layer of ink on the surface of the substrate, corresponding to the higher optical density. Reduction in the viscosity of the ink occurs only after the heat due to hysteresis loss has been generated, so that the differences in the optical density of the solid tone patches between the prints rendered by the $1 \times$ and $320 \times$ sleeves are smaller in the case of the 1.rthan the ones present at the 7.r.

\subsection{Trapping}

The difference in the values of trapping between the 1.r samples is so small that it could also have been in favour of the $1 \times$ sleeves. This means that the changes in the parameters impacting the trapping were not given enough time to show. Growth in the values of trapping was recorded in parallel with the increase in the number of printed rolls, especially in the case of the samples obtained using the $320 \times$ sleeves. The largest impact on this trend was made by the change in viscosity of the thin film of ink on the printing form, caused by the release of heat due to hysteresis loss. The impact made by the difference in viscosity of ink on the trapping is manifested through the transfer of a larger quantity of ink and the accelerated drying of the ink applied on the substrate. This results in trapping values which are closer by nature to trapping in the wet-to-dry printing, which is superior to the trapping in the wet-to-wet printing.

Another indicator of a reduction in the viscosity of the thin film of ink occurring during the printing of the $320 \times-$ 7.r is the less pronounced Saffman-Taylor instability. This instability is relatively noticeable at the samples obtained in all combinations, due to the high printing speed. The hydrodynamic defect (channels and larger holes in the layer of the over-printed ink), which occurs in the contact zone of the fluids is less pronounced. The differences in the viscosities of the fluids at the $320 \times-7 . r$ are still slightly smaller, compared to the $1 \times$ samples or the 1.r samples, regardless of the sleeves used.

\section{CONCLUSION}

The level of exploitation of the sleeves impacts the print parameters. While this impact improves some of these parameters, some other ones are affected adversely, and for some of them, the impact remains inconclusive. In addition 
to this, the impact made on the stability of the changes in the print quality parameters is also pronounced. Most typically, the change in print quality parameters occurs due to the impact of sleeve exploitation on other parameters of the printing process, or a change in the quality of the material. Considering that the print quality parameters vary and that their qualities are characterised in a different way, in order to achieve a good quality of a single parameter, it is recommended that a combination of print parameters be applied which are not necessarily favourable for the remaining quality parameters.

The main causes of the changes in the process parameters due to exploitation of the sleeves are the changed mechanical characteristics of their compressible layer. The process parameters affected by the change of qualities in the sleeves' compressible layer are the pressure and load placed on the printing form, as well as the resulting changes in quality of the form. Another parameter is the temperature of the thin film of ink on the printing elements, causing the change in ink viscosity, its transfer and drying before and inside the printing NIP.

Intensive exploitation of the sleeves leads to the following improvements or degradations in the print quality parameters and the process stability:

Deterioration in the print quality parameters:

- TVI, especially in highlights and lighter midtones.

- Doubling on $2 \%$ TV patches.

Improvement in parameters of the print quality:

- Better ink transfer, better penetration and drying, reduced spreading with the change of viscosity.

- Increase of optical density due to the reduction of resilience and increase of hardness of the printing formsleeve combination.

- Additional increase in the optical density due to the reduction in viscosity of the ink film.

- The pronounced increase in the trapping values occurring with the increase in the number of printed rolls, due to the changes in the ink viscosity.

- Less pronounced Saffman-Taylor instability due to the reduction in the differences between the viscosity of fluids (air-ink).

Deterioration in the process stability:

- $\quad$ Reduced possibility to compensate for the vibrations and bouncing.

- Change in the ink viscosity due to the heat released through hysteresis losses, and the consequences of the changing viscosity.

Improvements in the process stability:

- More stable TVI and smaller differences between the rolls, due to faster attainment of maximum residual strain.

Knowledge of the trend and intensity of changes caused by the exploitation of sleeves enables and makes their selection based on the printing requirements easier. Apart from that, the obtained knowledge could be used for determining in which cases the use of the sleeves with various level of exploitation is possible or even encouraged. Additionally, the quantification of changes opens the opportunities for their compensation during the digital setup for printing or during the very printing process. The characterisation of the impact made by sleeves on the parameters of quality of imprint on the paperboard graphic packaging also enables detecting the root cause of a number of occurrences and changes, while at the same time increasing the predictability of the product quality.

The advantage of the used methodology lies in the fact that it can be easily integrated into process control of the product on the industrial-scale production, using standard control patches and measuring devices. The methodology is not limited exclusively to the use of Twinlock sleeves, absorbent substrates, or cyan ink. However, with the use of other materials, different trends of changes in the parameters of the print quality are expected. These expectations further open the need to find new correlations of changes in process and print quality parameters caused by sleeve exploitation, which would take into account the specific properties of new materials and their interaction mechanisms.

Further research may be focused towards characterisation of the impact made by the exploitation of the sleeves upon the colourimetric values, uniformity of surfaces and defects in the shape of halftone dots.

\section{Acknowledgements}

This work was supported by the Serbian Ministry of Science and Technological Development, Grant No.: 35027 "The development of software model for improvement of knowledge and production in graphic arts industry."

\section{REFERENCES}

[1] Novaković, D., Dedijer, S., \& Mahović-Poljacek, S. (2010). A model for improving the flexographic printing plate making process. Technički vjesnik, 17(4), 403-410. Retrieved from https://hrcak.srce.hr/62914

[2] Tollenaar, D. \& Ernst, P. A. H. (1962).Optical density and ink layer thickness. In: W. H. Banks (Ed.), Advances in Printing Science and Technology 2, 214-234. Oxford, UK: Pergamon Press.

[3] Bould, D. C., Claypole, T. C., Bohan, M. F. J., \& Gethin, D. $\mathrm{T}$ (2004). Deformation of Flexographic Printing Plates. 56th TAGA Technical Conference, 146-162. Retrieved from https://www.researchgate.net/publication/265084813_Defor mation_of_Flexographic_Printing_Plates

[4] Bould, D. C., Claypole, T. C., \& Bohan, M. F. J. (2004). An investigation into plate deformation in flexographic printing. Proceedings of the Institution of Mechanical Engineers. Part B: Journal of Engineering and Manufacture, 218, 14991511. https://doi.org/10.1243/0954405042418428

[5] Mesic, B. (2006). Printability of polyethylene coated paper and paperboard (Doctoral dissertation). Karlstad University, Karlstad, SE, 11-19.

[6] Yang, L., Lenz, R., \& Kruse, B. (2001). Light Scattering and Ink Penetration Effect on Tone Reproduction. Journal of Optical Society of America, image science and vision, 18(2), 360-366. https://doi.org/10.1364/JOSAA.18.000360

[7] Zjakić I. (2007).Upravljanje kvalitetom ofsetnog tiska. Zagreb, HR: Hrvatska Sveučilišna naklada.

[8] Rabaud, M., Couder, Y., \& Michalland, S. (1991). Wavelength selection and transients in the one-dimensional array of cells of the printer's instability. European Journal of Mechanics-B/Fluids, 10(2), 253-260. Retrieved from http://www.fast.upsud.fr/ rabaud/Articles/Rabaud_1991.pdf

[9] Meyer, K. H., Durholz, R., \& Butterich, K. (1996). The printing plate as a quality characteristic in the reproduction of the printed image in flexographic printing. DFTA Technology Centre, Technical College of Printing, Stuttgart, DE 
[10] Holmvall, M. \& Uesaka, T. (2007). Nip Mechanics of Flexo Post-Printing on Corrugated Board. Journal of Composite Materials, 41(17), 2129-2145. https://doi.org/10.1177/0021998307074135

[11] Kilhenny, B. (2007). Restrain the Gain Cushions are Key to Control. Flexo, 32(8), 24.

[12] Hamblyn, A. (2015). Effect of plate characteristics on ink transfer in flexographic printing (Doctoral dissertation). Retrieved from https://cronfa.swan.ac.uk/Record/cronfa42827/Download/0 042827-02082018162525.pdf

[13] Petrović, S. \& Kašiković, N. (2018). Sleeve type influence on flexographic print quality. International Circular of Graphic Education and Research, (11), 35-51. Retrieved from

https://www.internationalcircle.net/international_circle/circ ular/issues/18_02/ICJ_11_2018_05

[14] Lee, B. (1998). Design characteristics unique to the flexographic printing process (MSc thesis). Rochester Institute of Technology, Rochester, New York. Retrieved fromhttps://pdfs.semanticscholar.org/db74/5edea4fa0bee003 003a2ab04c6b9c300ac86.pdf

[15] FTA (n.d.) Mounting Sleeves \& Bridges: Selecting the Correct Press Components for Increased Productivity. Retrieved from https://www.flexography.org/industrynews/mounting-sleeves-bridges-selecting-presscomponents-increase-productivity/

[16] Wolfram (2020). Analyze Bouncing Problems in a Printing Press. Retrieved from https://www.wolfram.com/systemmodeler/examples/industrial-manufacturing/configurationanalysis-cylinders.html

[17] Borbély, Á. \& Szentgyörgyvölgyi, R. (2011). Colorimetric Properties of Flexographic Printed Foils: the Effect of Impression. Óbuda University e-Bulletin, 2(1), 31-36.

[18] Bohan, M. F. J., Townsend, P., Hamblyn, S. M., Claypole, T. C., \& Gethin D. T. (2003).Evaluation of Pressures in Flexographic Printing. Proceedings of the TAGA 55th International Annual Technical Conference, 311-320. Retrieved from https://www.printing.org/tagaabstracts/t030311

[19] Valdec, D., Zjakić, I., \& Milković, M. (2013). The influence of variable parameters of flexographic printing on dot geometry of pre-printed printing substrate. Tehnički vjesnik, 20(4), 659-667.

[20] Hannah, M. (1951). Contact stress and deformation in a thin elastic layer. The Quarterly Journal of Mechanics and Applied Mathematics, 4(1), 94-105. https://doi.org/10.1093/qjmam/4.1.94

[21] Johnson, J., Rättö, P., Lestelius, M., \& Järnström, L. (2003). Dynamic Nip Pressure in a Flexographic Cl-Printing Press. 2003 TAGA Proceedings: 55th annual conference, Montreal, Quebec, 357-374. Retrieved from https://www.printing.org/taga-abstracts/t030357

[22] Fetsko, J. M. \& Walker, W. C. (1955). Measurement of Ink Transfer in Printing Coated Paper. American Ink Maker, 33(11), 38-44/67-69.

[23] Walker, W. C. \& Fetsko, J. M. (1955). A Concept of Ink Transfer in Printing. American Ink Maker, 33(12), 38-44/6971.

[24] Davies, G. R. \& Claypole, T. C. (2006). Effect of Viscosity on Ink Transfer in Gravure Printing. iarigai, Leipzig, DE.

[25] Zang, Y. H. (1992). Asymmetric splitting and ink transfer: a new ink transfer model. Proceedings of the 6th International Printing and Graphic Arts conference TAPPI, Peachtree Corners, Georgia, 103-112.

[26] Dejidas, L. \& Destree, T. (2005) Sheetfed Offset Press Operating. Pittsburgh, Pennsylvania: PIA/GATF Press.

[27] Hsu, B. (1962).Some Observations on the Ink-Paper Relationship During Printing. Advances in Printing Science and Technology, 2, Problems in High Speed Printing. New York, NY:Pergamon Press, 1-10.

[28] Fernández, M., Muñoz, M. E., \& Santamaría, A. (1998). Rheological Analysis of Highly Pigmented Inks: Flocculation at High Temperatures. Journal of Rheology, 42(2), 239-253. https://doi.org/10.1122/1.550948

[29] Rentzhog, M. \& Fogden, A. (2005). Rheology and Surface Tension of Water-Based Flexographic Inks and Implications for Wetting of PE-Coated Board. Nordic Pulp and Paper Research Journal, 20(4), 399-409. https://doi.org/10.3183/nppri-2005-20-04-p399-409

[30] Nordström, J.-E. P. \& Johnson, J. (2002).CSWO (Cold Set Waterless Web Offset) Using Standard Tmp/Dip Newsprint. International Printing \& Graphic Arts Conference, Bordeaux, France, 11.

[31] Olsson, R., Yang, L., van Stam, J., \& Lestelius, M. (2007). Effects of elevated temperature on flexographic printing. In: N. Enlund, M. Lovreček (Eds.). Advances in Printing and Media Technology. Proceedings of the 34th International Research Conference of iarigai, Grenoble, France, 85-93.

[32] Cozzens, S. L., Butto, A. M., Schaeffer, W. D., \& Zettlemoyer, A. C. (1965). Ink Penetration During HighSpeed Printing of Uncoated Paper. Proceedings of the Eighth International Conference of Printing Research Institutes, Aulanko, Finland, 1-24. https://doi.org/10.1016/B978-0-08-012289-2.50006-5

[33] FlexoTech (2019). New hardness level for Flint Group's rotec Compressible Sleeve. FlexoTech, Whitmar Publications. Retrieved from

https://www.flexotechmag.com/news/18958/new-hardnesslevel-for-flint-group-s-rotec-compressible-sleeve/

[34] Qi, H. J. \& Boyce, M. C. (2005). Stress-strain behavior of thermoplastic polyurethanes. Mechanics of Materials, 37(8), 817-839. https://doi.org/10.1016/j.mechmat.2004.08.001

[35] International Organization for Standardization. (2005). Rubber, vulcanized or thermoplastic-Determination of stress relaxation in compression at ambient and at elevated temperatures (ISO Standard No. 3384:2005).

\section{Contact information:}

Saša PETROVIĆ, Teaching assistant

(Corresponding author)

Faculty of Technical Sciences, University of Novi Sad,

Trg Dositeja Obradovića 6, 21000 Novi Sad, Serbia

E-mail: petrovic.sasa@uns.ac.rs

Nemanja KAŠIKOVIĆ, Associate professor

Faculty of Technical Sciences, University of Novi Sad

Trg Dositeja Obradovića 6, 21000 Novi Sad, Serbia

E-mail: knemanja@uns.ac.rs

Dragoljub NOVAKOVIĆ, Full professor

Faculty of Technical Sciences, University of Novi Sad,

Trg Dositeja Obradovića 6, 21000 Novi Sad, Serbia

E-mail: novakd@uns.ac.rs

Gordana BOŠNJAKOVIĆ, Teaching assistant

Faculty of Technical Sciences, University of Novi Sad,

Trg Dositeja Obradovića 6, 21000 Novi Sad, Serbia

E-mail: gordana.delic@uns.ac.rs

Emine ARMAN KANDIRMAZ, Research assistant

School of Applied Sciences, Marmara University,

Goztepe, 34722 Istanbul, Turkey

E-mail: earman@marmara.edu.tr

Arif OZCAN, Associate professor

School of Applied Sciences, Marmara University,

Goztepe, 34722 Istanbul, Turkey

E-mail: arifozcan@marmara.edu.tr 